\title{
Desenvolvimento de uma Lista de Verificação em Comunicação e Linguagem para os Transtornos do Espectro Autístico
}

\author{
Silvana Batista Gaino1 - Universidade Federal do Recôncavo da Babia, Santo Antônio de Jesus, Brasil \\ Manoel Henrique Salgado - Universidade Estadual Paulista, Bauru, Brasil \\ Fernanda Dreux Miranda Fernandes - Universidade de São Paulo, São Paulo, Brasil
}

\begin{abstract}
Resumo
A Lista de Verificação em Comunicação e Linguagem aqui apresentada foi elaborada para avaliar as especificidades de dois grupos distintos: os transtornos do espectro autístico e outras alterações de desenvolvimento da linguagem. Com essa finalidade foi aplicada em 60 participantes, divididos em dois grupos: 20 portadores de transtornos do espectro autístico $(M=7,11)$ e 40 portadores de outras alterações de desenvolvimento da linguagem $(M=4,10)$. Foi utilizado o teste do Qui-Quadrado para avaliar a validade do conteúdo e determinar quais questões deveriam ser retiradas do instrumento por não serem significativas. Os outros testes utilizados foram o Índice de Concordância Kappa e o teste de Mann-Withney. A Lista também apresentou uma sensibilidade de $90 \%$ e uma especificidade de 97,5\%, o que demonstra a viabilidade de sua utilização como instrumento auxiliar na detecção de transtornos do espectro autístico.

Palavras-chave: Lista de verificação, Comunicação, Linguagem, Transtornos do espectro autístico.
\end{abstract}

\section{Development of a Checklist for Communication and Language for the Autistic Spectrum Disorders}

\begin{abstract}
The Checklist in Communication and Language presented herein was elaborated to assess specificities of two distinct groups: the autistic spectrum disorders and other languages development alterations. With this objective, it was applied in 60 participants divided into two groups: 20 subjects with autistic spectrum disorders $(M=7,11)$ and 40 with language development alterations $(M=4,10)$. The statistical analysis held after application was the Chi-Square distribution test to assess the content validity and to determine what questions should be removed of the instrument for not being significant. The other tests used were the Kappa Index Agreement and Mann-Whitney test. The Checklist indicated sensibility of $90 \%$ and specificity of $97,5 \%$, which demonstrates viability to be used as an auxiliary tool in detecting the autistic spectrum disorders.

Keyword: Checklist, Communication, Language, Autistic spectrum disorders.
\end{abstract}

Muitos são os estudos sobre o processo de aquisição da linguagem, bem como as suas concepções. Alguns vão valorizar a forma, outros a ação comunicativa e outros o conteúdo. Autores como Bates, Camaione e Volterra (1976), Brunner (1975, 1978), Bullowa (1979), Bateson (1979), Chappell e Sander (1979), Brazelton (1979) e Harding (1983), por exemplo, valorizaram nos seus estudos as trocas comunicativas da criança no período pré-verbal e afirmaram sua importância para a aquisição da linguagem.

Já Bloom e Lahey (1988) consideraram nos seus estudos as três dimensões da linguagem: forma, conteúdo e uso. A forma inclui a fonologia e a morfossintaxe; o conteúdo, o léxico e a semântica das palavras e locuções; e o uso, as habilidades pragmáticas, como as regras convencionais e as funções da comunicação.

Battes e cols. (1976) afirmam que entender como uma criança aprende a se comunicar não é suficiente para entender como ela aprende a língua

${ }^{1}$ Endereço para correspondência:

Rua do Cajueiro, s./n. - Cajueiro - 44574-490

Santo Antônio de Jesus-BA

E-mail: silgaino@hotmail.com propriamente dita. Hage (1997) relatou, em seus trabalhos, que apesar de ainda não ser possível desvendar completamente a construção da língua materna, todos os trabalhos desenvolvidos nessa área são de um valor inestimável por auxiliarem na análise da linguagem e mostrarem que ela não se restringe à sua forma. Para esta autora a comunicação pré-verbal, embora não seja condição única para o aparecimento da linguagem enquanto estrutura, invariavelmente a precede, e os estudos empíricos que provam este fato fornecem subsídios para a avaliação de crianças que não falam numa idade cronológica posterior ao esperado.

Muitos ainda são os questionamentos existentes a respeito dos fatores que influenciam nesse processo de aquisição da linguagem e enquanto alguns autores afirmam que o desenvolvimento social $\mathrm{e}$ cognitivo são pré-requisitos, outros vão afirmar que essas áreas, na verdade, são afetadas pelo desenvolvimento da linguagem. Isso leva à consideração de que na realidade o que existe é uma interdependência entre todos esses aspectos (MoliniAvejonas \& Fernandes, 2004).

Fernandes (1995, 1996, 2000) propõe, então, uma forma de análise funcional da linguagem, a partir de uma perspectiva pragmática, que inclui funções 
comunicativas, identifica atos comunicativos expressos por minuto e o meio comunicativo utilizado (verbal, vocal e gestual). Estas funções são divididas em mais interativas (pedido de objeto, pedido de ação, pedido de rotina social, pedido de consentimento, pedido de informação, protesto, reconhecimento do outro, comentários, nomeação, exclamativa, narrativa, jogo compartilhado, expressão de protesto e exibição) e menos interativa (autorregulatória, performativa, reativa, não focalizada, jogo e exploratória) (Fernandes, 2002). Baseando-se nessa perspectiva, o estudo da linguagem deve considerar a relação entre linguagem e os contextos linguísticos e não linguísticos e os fatores ambientais e sociais (Fernandes, 1996, 1998; Ochs Keenan \& Schieffelin, 1983).

Para Fernandes (2002), a linguagem, durante o seu desenvolvimento, vai envolver os aspectos orgânicos, emocionais, cognitivos e sociais e só vai ter significado em relação ao contexto no qual se manifesta, e qualquer investigação ou intervenção terapêutica da linguagem deve considerar esses elementos. Essa interdependência não é verdadeira apenas para o desenvolvimento típico, mas também para outras patologias que se manifestam na infância. Dentre estas, uma das que vêm sendo mais estudadas na atualidade e que permanecem intrigantes sob vários aspectos são os transtornos do espectro autístico. A primeira descrição desse transtorno foi realizada por Kanner em 1943 e, desde então, inúmeros pesquisadores dedicam-se ao seu estudo apontando, atualmente, para uma base biológica e um forte componente genético (Farrell, 2008).

Embora existam diferentes linhas de pesquisa, bem como diferentes explicações sobre as prováveis áreas afetadas pelo autismo, os aspectos linguísticos aparecem como uma marca constante em todos os estudos que analisam esse quadro (Fernandes, 2000a). Portanto, fica evidente que os questionamentos sobre o desenvolvimento da cognição, da socialização e da linguagem são absolutamente importantes para uma compreensão mais adequada dos transtornos do espectro autístico.

Atualmente uma das teorias cognitivas utilizadas para se pensar o autismo advém dos trabalhos de Frith (1988, 1989) e Baron-Cohen (1988, 1990, 1991), que questionam o conceito primitivo de Kanner e a própria noção de psicose. Baron-Cohen (1988) apresenta a teoria da metarrepresentação, considerando como fundamental para a compreensão do quadro a incapacidade do autista de compreender os estados mentais do outro. Assim sendo, os déficits pragmáticos de relacionamento social e de linguagem seriam dela decorrentes. Dessa forma, pensar o autismo dentro de uma visão cognitiva é uma possibilidade capaz de permitir sua compreensão dentro de um modelo teórico.

Utilizando a Teoria da Mente como referencial, Carvalho, Mecca e Lichtig (2008) desenvolveram uma pesquisa sobre as relações da linguagem e essa teoria em 100 crianças com idades entre 7 e 8 anos, concluindo que por volta dos 7 anos a maioria delas já adquiriu a Teoria da Mente. Os autores afirmaram que a interação e a utilização de elementos abstratos durante a conversação podem influenciar $O$ desempenho das crianças nas tarefas relacionadas à verificação da aquisição da Teoria da Mente.

Com a pretensão de verificar o perfil funcional da comunicação de crianças e adolescentes com transtornos do espectro autístico, Fernandes e Miilher (2008) desenvolveram uma pesquisa, com 117 participantes, com idades variando entre 2 a 16 anos, utilizando o Autism Behavior Checklist (ABC). Os resultados indicaram a existência de correlações significativas entre a pontuação no $\mathrm{ABC}$ e os resultados dos perfis funcionais da comunicação, com exceção da subescala de linguagem. Diante desses resultados, as autoras sugeriram que $\mathrm{o} A B C$ não deve ser utilizado como único recurso para o diagnóstico dessa população.

Num outro estudo, que teve como objetivo principal investigar o perfil comunicativo de crianças com diagnóstico de distúrbio do espectro autístico (DEA), de transtorno do déficit de atenção com hiperatividade (TDAH) e de distúrbio específico de linguagem (DEL), com base nas respostas dos pais a um questionário - Children's Communication Checklist-2 (CCC-2) - verificou-se que as crianças com DEA possuem atraso de linguagem em diversas áreas, sendo a pragmática a mais evidente delas. Esses autores consideram que o DEL é uma patologia primária que acomete o desenvolvimento linguístico, cujas dificuldades recaem principalmente nos aspectos estruturais, mas também são observados déficits pragmáticos, que normalmente decorrem desse déficit linguístico (Befi-Lopes \& Cáceres, 2010).

Esse trabalho corroborou as afirmações de Bishop (1989) de que são muitas as dificuldades de linguagem observadas numa criança com autismo, incluindo seus aspectos sociais e formais. Esses aspectos se configuram como um sintoma significativo e como um referencial para o prognóstico do quadro (Amato, 2000; Tamanaha, Perissinoto \& Chiari, 2008).

Considerando as afirmações teóricas acima relatadas e as discussões atuais sobre o diagnóstico do autismo infantil, que pontuam a necessidade de instrumentos que avaliem áreas específicas comprometidas por este transtorno, o objetivo do presente trabalho foi elaborar uma Lista de Verificação 
em Comunicação e Linguagem para Transtornos do Espectro Autístico, aplicá-la num projeto piloto e depois, utilizando o ABC, fazer uma comparação dos resultados alcançados pelos participantes. A lista elaborada deveria considerar os aspectos do desenvolvimento da linguagem e da sua utilização nas tentativas de comunicação de modo a oferecer material sistematizado na avaliação de tais aspectos e que fosse passível de ser utilizado em investigações futuras. Escalas como a ATA, o ABC, o ADOS, o ADI-R e o CHAT, que já foram traduzidas e adaptadas e possuem estudos de validade e precisão no Brasil, consideram os aspectos de comportamento e de desenvolvimento de uma forma geral, não tendo como objetivo uma avaliação de aspectos mais específicos, como os da linguagem, o que vem sendo uma tendência mundial na atualidade, verificada principalmente em congressos internacionais.

Vale ressaltar que este instrumento pode vir a ser uma medida válida para diferenciar as crianças com desordens que acometem o desenvolvimento da linguagem daquelas que apresentam desenvolvimento típico da mesma, podendo ser utilizado como instrumento de triagem, e o estudo descrito apresenta os primeiros resultados de uma investigação em contexto brasileiro.

\section{Método}

\section{Participantes}

A amostra foi composta por 60 participantes de faixa etária entre 3 e 13 anos, divididos em dois grupos: o grupo de pesquisa (GA) e o grupo controle (GB). O GA foi composto por 20 portadores de diagnóstico de transtorno do espectro autístico, com média de idade de 7a 11m (DP=2,10), diagnosticados por dois psiquiatras infantis, baseados nos critérios do DSM-IV-TR, de ambos os sexos, filmados por 20 minutos. Eles recebiam atendimentos regulares por equipes interdisciplinares.
Os 40 participantes do GB, de ambos os sexos, com média de idade de 4a 10m (DP=1, 2), eram portadores de alterações de linguagem não decorrentes de perda sensorial e/ou distúrbio afetivo-cognitivo e já haviam sido submetidos a avaliação audiométrica, fonoaudiológica, psicológica e neurológica. Estes eram atendidos semanalmente, por estagiárias do curso de Fonoaudiologia da UNESP/Marília-SP, e também foram filmados por 20 minutos.

A utilização de um grupo controle (GB) de participantes com outras alterações de linguagem não decorrentes de perda sensorial e/ou distúrbio afetivocognitivo, no qual foram aplicados os mesmos instrumentos, foi proposta para a comparação dos dados obtidos com o grupo de portadores de transtornos do espectro autístico e para tentativa de diferenciação dos comprometimentos do desenvolvimento da linguagem apresentados pelos dois grupos (GA).

\section{Instrumentos}

\section{Lista de verificação}

É composta por 29 itens dicotômicos, com opção de resposta Sim ou Não (Tabela 1), que visam avaliar as manifestações relacionadas à comunicação e linguagem e que os avalia nos seguintes aspectos: funcionalidade, conteúdo e forma. Esses itens constam de instrumentos já existentes, que avaliam aspectos gerais do desenvolvimento e que são utilizados para avaliação e diagnóstico dos transtornos do espectro autístico, porém nenhum dos estudados para esta pesquisa abrangeu todos nem apresentava como objetivo principal avaliar a dimensão específica do desenvolvimento da comunicação e da linguagem, abarcando também outras dimensões que não foram foco deste estudo.

Tabela 1 - Lista de Verificação em Comunicação e Linguagem para Transtornos do Espectro Autístico (Continua)

\begin{tabular}{|c|c|c|c|}
\hline \multirow{2}{*}{\multicolumn{4}{|c|}{$\begin{array}{l}\text { Características Observadas } \\
\text { 1-Executa ordens complexas }\end{array}$}} \\
\hline & & & \\
\hline \multicolumn{4}{|l|}{ 2-Faz pedidos de objetos } \\
\hline \multicolumn{4}{|l|}{ 3- Aponta para um objeto quando solicitado } \\
\hline \multicolumn{4}{|l|}{ 4-Usa a primeira pessoa do singular (eu, me, meu, mim, comigo) } \\
\hline \multicolumn{4}{|l|}{ 5-Pede ajuda ou conforto } \\
\hline \multicolumn{4}{|l|}{ 6-Faz nomeações } \\
\hline \multicolumn{4}{|l|}{ 7-Faz contato ocular } \\
\hline \multicolumn{4}{|l|}{ 8-Produz sons ininteligíveis } \\
\hline \multicolumn{4}{|l|}{ 9-Faz comentários } \\
\hline 10-Interrompe a atividade quando ouve não & & & \\
\hline
\end{tabular}


Tabela - Lista de Verificação em Comunicação e Linguagem para Transtornos do Espectro Autístico

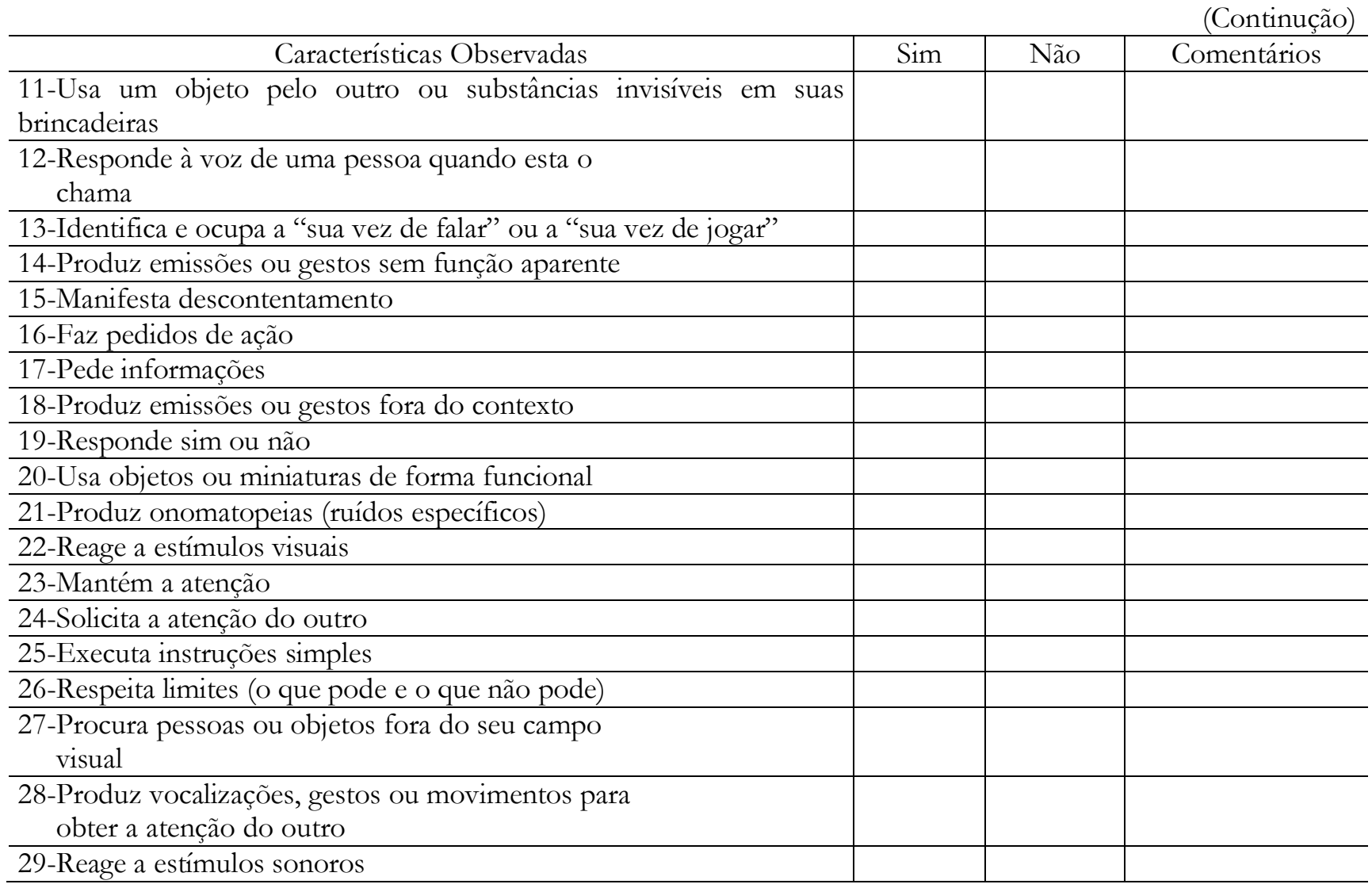

Os itens que compõem a referida lista de verificação são originários dos seguintes instrumentos: 1) Executa ordens complexas - CHAT/ADOS; o item 2) Faz pedidos de objetos - CARS/ADOS; o item 3) Aponta para um objeto quando solicitado CHAT/ADI-R; o item 4) Usa a primeira pessoa do singular (eu, me, meu, mim, comigo) - CARS/ADI-R; 5) Pede ajuda ou conforto - CHAT/ADI-R; 6) Faz nomeações - CARS/ADOS; 7) Faz contato ocular CHAT/ADOS; 8) Produz sons ininteligíveis ABC/ADI-R; 9) Faz comentários - CARS/ADOS; 10) Interrompe a atividade quando ouve não CARS/ADOS; 11) Usa um objeto pelo outro ou substâncias invisíveis em suas brincadeiras CHAT/ADOS/ADI-R; 12) Responde à voz de uma pessoa quando esta o chama - CHAT/ADOS; 13) Identifica e ocupa a "sua vez de falar" ou a "sua vez de jogar" - CARS/ADOS; 14) Produz emissões ou gestos sem função aparente - ABC/ADI-R; 15) Manifesta descontentamento - CARS/ADOS; 16) Faz pedidos de ação - CARS/ADI-R; 17) Pede informações CARS/ADOS; 18) Produz emissões ou gestos fora do contexto - ABC/ADI-R; 19) Responde sim ou não CHAT/ADOS; 20) Usa objetos ou miniaturas de forma funcional - CHAT/ADOS-G/ADI-R; 21) Produz onomatopeias (ruídos específicos) -
CHAT/ADI-R; 22) Reage a estímulos visuais - CARSADI-R; 23) Mantém a atenção - CHAT/ADOS; 24) Solicita a atenção do outro - CHAT/ADOS; 25) Executa instruções simples - CHAT/ADOS; 26) Respeita limites (o que pode e o que não pode) CARS/ADI-R; 27) Procura pessoas ou objetos fora do seu campo visual - CHAT/ADOS/ADI-R; 28) Produz vocalizações, gestos ou movimentos para obter a atenção do outro - CARS/ADOS e 29) Reage a estímulos sonoros - CHAT e ADI-R.

Numa primeira etapa deste estudo preliminar, depois de elaborada a lista, a pesquisadora assistiu às filmagens dos 60 participantes da pesquisa e preencheu, para cada um deles uma cópia da lista de verificação, numerou as dos portadores de transtornos do espectro autístico de 1 a 20 e as dos portadores de outras alterações da linguagem de 21 a 60, misturou aleatoriamente todas as cópias, procedeu a um sorteio e agrupou-as em envelopes que foram numerados. Em cada um dos envelopes 1, 2, 3 e 4 foram colocadas 8 cópias da lista de verificação preenchidas pela pesquisadora. Já nos envelopes 5, 6, 7 e 8 foram colocadas 7 cópias da lista de verificação preenchidas pela pesquisadora.

Logo em seguida, os envelopes pares foram entregues para 4 fonoaudiólogas, que desempenhariam 
o papel de juízes, e foi solicitado que avaliassem as cópias das listas contidas no seu envelope, já preenchidas e fizessem a escolha do diagnóstico que melhor descrevesse o caso apresentado. $\mathrm{Na}$ parte posterior de cada lista eram apresentadas as opções: 1) ( ) Alterações do desenvolvimento da linguagem ou 2) ( ) Transtornos do espectro autístico. Os envelopes ímpares foram entregues para 4 psicólogas, que também desempenhariam o papel de juízes, e foi solicitado que realizassem a mesma avaliação que foi solicitada às fonoaudiólogas e que também apontassem o diagnóstico que melhor descrevesse cada caso.

$\mathrm{Na}$ segunda etapa deste estudo preliminar, realizada seis meses depois da primeira, foram novamente numeradas 60 cópias da lista de verificação (as de 1 a 20 eram para transtornos do espectro autístico e as de 21 a 60 eram para outras alterações da linguagem) e as filmagens correspondentes a cada participante também foram numeradas, utilizando-se os mesmos critérios (as filmagens dos participantes com transtornos do espectro autístico foram numeradas de 1 a 20 e as dos portadores de outras alterações de linguagem de 21 a 60). Procedeu-se a um novo sorteio e nesta etapa as listas foram encaminhadas para os juízes em branco, juntamente com a filmagem correspondente do participante (por ex: para a lista $\mathrm{n}^{\circ} 8$, a filmagem a ser assistida seria do participante $n^{\circ} 8$ ). Depois de assistir a filmagem de cada participante, contida em seu envelope, cada juiz deveria preencher a cópia da lista correspondente e fazer a escolha do diagnóstico que melhor descrevesse cada caso apresentado. As mesmas opções apresentadas na primeira etapa foram apresentadas nessa etapa. As fonoaudiólogas novamente ficaram com os envelopes pares e as psicólogas com os ímpares.

Durante a primeira etapa, todos os envelopes foram recolhidos 3 dias depois que haviam sido entregues. $\mathrm{Na}$ segunda, 1 semana depois, e deve-se salientar aqui que os juízes que participaram desta pesquisa não mantiveram contato entre si, a maioria morando em cidades ou bairros distintos, e não receberam qualquer devolutiva sobre os resultados antes do final da pesquisa.

A Lista de Verificação aqui apresentada em nenhum momento se propõe a realizar por si só o diagnóstico de um quadro tão complexo e deve ter seus resultados analisados especificamente como uma triagem. Pode ser preenchida pelos profissionais responsáveis pelo processo de triagem, utilizando como referenciais suas observações e os dados coletados junto ao(s) responsável(is) pelo paciente.

Autism Behavior Checklist (ABC)
$\mathrm{O} A B C$ é uma listagem de comportamentos não adaptativos traduzida e pré-validada para a Língua Portuguesa, que busca identificar crianças pertencentes ao espectro autístico. Permite a descrição detalhada das características comportamentais atípicas de cada indivíduo. Utiliza pontuações balanceadas (faixa de $1 \mathrm{a}$ 4 pontos), que vão variar de acordo com a ocorrência de cada comportamento na patologia. Contempla diversas áreas do desenvolvimento: sensorial (9), relacional (12), Uso do corpo e objeto (12), Linguagem (13) e Pessoal- Social (11) (Tamanha, Marteleto e Perissinoto, 2008).

Após sua aplicação, um perfil comportamental é traçado com a finalidade de avaliar a severidade da patologia. Uma pontuação igual a 68 tem sido considerada como de alta probabilidade para a classificação do autismo infantil. Entre 67 e 54 pontos, há moderada probabilidade de classificação e entre 53 e 47 pontos, a probabilidade é baixa.

$\mathrm{O} A B C$ foi escolhido para ser utilizado nesta pesquisa piloto porque, na época que a mesma foi desenvolvida (2003), ele era o único que havia sido utilizado como referencial para a construção da lista de verificação que já era traduzido e que tinha estudos de validade sendo propostos no Brasil. Ele foi aplicado nos 60 participantes deste estudo $(\mathrm{GA}=20$ e $\mathrm{GB}=40)$ pela pesquisadora, numa última etapa, após ter assistido as filmagens dos participantes.

\section{Procedimento}

Nas filmagens que foram realizadas durante sessões de atendimento fonoaudiológico e psicológico foram utilizados brinquedos que já eram conhecidos pelos participantes, e que eram normalmente utilizados nas sessões de atendimento, como por exemplo: miniaturas de animais, carrinhos, bonecos, etc. Após as filmagens os participantes foram avaliados pela pesquisadora, que possui experiência na área de avaliação e atendimento em Transtornos Invasivos do Desenvolvimento, e por oito profissionais, com até 2 anos de formados, que não possuíam experiência específica na área dos transtornos invasivos do desenvolvimento e que desempenharam o papel de juízes, sendo 4 fonoaudiólogas e 4 psicólogas.

Numa etapa posterior, a pesquisadora assistiu às filmagens, que foram realizadas pelas estagiárias responsáveis pelos atendimentos dos participantes da pesquisa e, com base na observação das filmagens, preencheu as listas de verificação. Depois comparou a sua avaliação com a realizada pelos psiquiatras e, uma vez que as mesmas apresentavam concordância, separou as listas de verificação correspondentes aos portadores de transtornos do espectro autístico das dos portadores de alterações do desenvolvimento da 
linguagem. Numerou as GA de 1 a 20 e as do GB de 21 a 60. Procedeu então ao sorteio, dividindo as listas, já preenchidas, em envelopes pares e ímpares, que foram enviados para os juízes, que receberam a orientação de optar por uma das seguintes alternativas de diagnóstico para cada criança: 1) Transtorno do Espectro Autístico (TEA) ou 2) Alterações de Linguagem (AL).

Seis meses depois da primeira coleta de dados, as listas de verificação foram novamente numeradas e separadas, de acordo com os mesmos critérios utilizados na etapa anterior, porém desta vez as mesmas não foram preenchidas. Os juízes, que já haviam participado da etapa anterior, deveriam ler as instruções contidas no envelope e no cabeçalho da lista de verificação e: 1) preencher as cópias numeradas, após assistirem os 20 minutos das filmagens de cada criança, assinalando as características que foram observadas; 2) após esse preenchimento, deveriam escolher a opção de diagnóstico que, a seu ver, melhor descrevesse o quadro apresentado por essa criança.

\section{Resultados e discussão}

Quando os juízes terminavam suas avaliações, os dados coletados eram analisados, considerando-se as seguintes variáveis: o índice de concordâncias e discordâncias, o levantamento das questões que tiveram um peso maior na caracterização do quadro de transtornos do espectro autístico e o levantamento das questões que tiveram um peso maior na caracterização do quadro de alterações de desenvolvimento da linguagem. Com base nesses dados, foram calculados a média e o percentual de acertos dos juízes, registrando-se o número total de vezes que cada questão foi assinalada e a escolha entre sim ou não de cada questão.

Para finalizar, como não existiam instrumentos para avaliar aspectos específicos do desenvolvimento da comunicação e da linguagem relacionados aos transtornos do espectro autístico, optou-se pela aplicação do Autism Behavior Checklist (ABC) (Marteleto, 2003) nos participantes do estudo, para verificar se os dados levantados pela Lista de Verificação, que nortearam a escolha das hipóteses diagnósticas dos juízes, seriam suficientes e adequados.

As questões da Lista de Verificação avaliaram aspectos do desenvolvimento da comunicação e da linguagem, como os de funcionalidade (por exemplo: 3 , 7,15 ); conteúdo (por exemplo: 1, 20, 25); e forma (por exemplo: 10, 22, 29). Segundo Hage (1997) todos os trabalhos que abordem o desenvolvimento da linguagem são de um valor inestimável por auxiliarem na análise do desenvolvimento da mesma e por mostrarem que ela não se restringe à sua forma. Para esta autora a comunicação pré-verbal, embora não seja condição única para o aparecimento da linguagem enquanto estrutura, invariavelmente a precede, e os estudos empíricos que provam este fato fornecem subsídios para a avaliação de crianças que não falam numa idade cronológica posterior ao esperado.

Para o levantamento do número de concordâncias e discordâncias, e posterior comparação entre as etapas do estudo, foi realizado o levantamento das respostas dadas pela pesquisadora e pelos juízes. $\mathrm{Na}$ avaliação do GA, por meio da lista de verificação, evidenciou-se uma discordância superior à concordância entre as respostas dadas pela pesquisadora e os juízes, para os participantes 10,16 e 17 , que nas suas avaliações receberam como hipótese diagnóstica autismo não especificado. Decidiu-se, então, pela exclusão desses participantes do GA. Considerando-se, então, os 17 participantes que permaneceram no Grupo A, a Tabela 2 mostra o total de concordâncias, discordâncias e a porcentagem de concordância em relação ao critério não observado, que foi incluído pelos juízes, na segunda etapa, quando eles tiveram que, primeiramente avaliar os participantes, para depois optar pelo diagnóstico que melhor os descrevesse, utilizando as filmagens que haviam sido realizadas.

Tabela 2 - Apresentação dos índices de concordância e discordância do Grupo A, nas duas etapas de avaliação

(Continua)

\begin{tabular}{ccccc}
\hline Questões & $\begin{array}{c}\text { Concordância } \\
\text { TEA }\end{array}$ & $\begin{array}{c}\text { Discordância } \\
\text { TEA }\end{array}$ & $\begin{array}{c}\text { Não Observado } \\
\text { TEA }\end{array}$ & \% de Concordância \\
\hline 1 & 12 & 2 & 3 & 86 \\
2 & 17 & 0 & 0 & 100 \\
3 & 13 & 4 & 0 & 76 \\
4 & 15 & 2 & 0 & 88 \\
5 & 12 & 3 & 2 & 80 \\
6 & 16 & 1 & 0 & 94 \\
7 & 14 & 2 & 1 & 88 \\
8 & 12 & 4 & 1 & 75 \\
\hline
\end{tabular}


Tabela 2 - Apresentação dos índices de concordância e discordância do Grupo A, nas duas etapas de avaliação

\begin{tabular}{|c|c|c|c|c|}
\hline Questões & $\begin{array}{c}\text { Concordância } \\
\text { TEA }\end{array}$ & $\begin{array}{c}\text { Discordância } \\
\text { TEA }\end{array}$ & $\begin{array}{c}\text { Não Observado } \\
\text { TEA }\end{array}$ & \% de Concordância \\
\hline 9 & 9 & 8 & 0 & 53 \\
\hline 10 & 13 & 4 & 0 & 76 \\
\hline 11 & 14 & 3 & 0 & 82 \\
\hline 12 & 15 & 1 & 1 & 94 \\
\hline 13 & 7 & 6 & 4 & 54 \\
\hline 14 & 7 & 10 & 0 & 41 \\
\hline 15 & 17 & 0 & 0 & 100 \\
\hline 16 & 13 & 4 & 0 & 76 \\
\hline 17 & 4 & 13 & 0 & 24 \\
\hline 18 & 14 & 2 & 1 & 88 \\
\hline 19 & 16 & 1 & 0 & 94 \\
\hline 20 & 11 & 3 & 3 & 79 \\
\hline 21 & 10 & 7 & 0 & 59 \\
\hline 22 & 15 & 2 & 0 & 88 \\
\hline 23 & 13 & 2 & 2 & 87 \\
\hline 24 & 1 & 13 & 3 & 7 \\
\hline 25 & 7 & 8 & 2 & 47 \\
\hline 26 & 7 & 8 & 2 & 47 \\
\hline 27 & 8 & 8 & 1 & 50 \\
\hline 28 & 9 & 8 & 0 & 53 \\
\hline 29 & 14 & 3 & 0 & 82 \\
\hline 30 & 7 & 8 & 2 & 47 \\
\hline 31 & 9 & 7 & 1 & 56 \\
\hline 32 & 9 & 8 & 0 & 53 \\
\hline 33 & 9 & 8 & 0 & 53 \\
\hline
\end{tabular}

A partir da análise das questões, com base nas discordâncias entre a pesquisadora e os juízes, e utilizando como referencial para o critério de corte os índices abaixo de 50\%, pudemos observar que as questões 14, 17, 24, 25, 26 e 30 não apresentaram uma boa capacidade de identificação de sinais de transtornos do espectro autístico. Essa diferença significativa na avaliação pode ser justificada pelo fato de os juízes não terem sido treinados antes da avaliação e terem utilizado somente os conhecimentos que já possuíam e que não eram específicos da área dos transtornos psiquiátricos da infância.

O critério de corte de $50 \%$ foi escolhido com base nos critérios de corte dos instrumentos que foram utilizados como referências no presente estudo.

Quando comparadas as respostas sim e não, no GA, na primeira etapa do estudo, pôde-se observar um percentual maior de não em relação ao sim, ou seja, os juízes concordavam entre si que os participantes do GA não apresentavam o desenvolvimento da comunicação ou da linguagem apontados pelo item. A prevalência da resposta não em comparação ao sim possibilitou a identificação de sinais de transtornos do espectro autístico. Já na segunda etapa do estudo, observou-se que novamente prevalece um percentual maior de não em relação ao sim, mesmo com a inclusão da categoria do não observado, o que corroborou o fato anteriormente levantado de que a lista de verificação pode identificar sinais de transtornos do espectro autístico.

$\mathrm{Na}$ avaliação final das questões no GA, notou-se que duas questões tiveram $100 \%$ de concordância; três questões tiveram de 80 a $90 \%$ de concordância; nove questões tiveram de 70 a $80 \%$ de concordância; cinco questões tiveram de 60 a $70 \%$ de concordância; oito questões tiveram de 50 a $60 \%$ de concordância. E na avaliação do GB mediante a lista de verificação, evidenciou-se uma discordância superior à concordância para os participantes 25 e 58 entre as respostas dadas pela pesquisadora e os juízes. No caso dos participantes 36, 39, 50, 52 e 54 a concordância foi inferior à soma da discordância e do não observado. 
Decidiu-se, então, pela exclusão desses participantes do

GB.

Tabela 3 - Apresentação dos índices de concordância e discordância do Grupo B, nas duas etapas de avaliação

\begin{tabular}{|c|c|c|c|c|}
\hline Questões & Concordância AL & Discordância AL & Não Observado AL & \% de Concordância \\
\hline 1 & 19 & 5 & 9 & 83 \\
\hline 2 & 20 & 12 & 1 & 65 \\
\hline 3 & 22 & 8 & 3 & 76 \\
\hline 4 & 16 & 14 & 3 & 57 \\
\hline 5 & 30 & 1 & 2 & 97 \\
\hline 6 & 19 & 13 & 1 & 59 \\
\hline 7 & 13 & 16 & 4 & 46 \\
\hline 8 & 21 & 11 & 1 & 69 \\
\hline 9 & 32 & 0 & 1 & 99 \\
\hline 10 & 12 & 18 & 3 & 43 \\
\hline 11 & 17 & 15 & 1 & 53 \\
\hline 12 & 23 & 6 & 4 & 83 \\
\hline 13 & 18 & 13 & 2 & 61 \\
\hline 14 & 31 & 1 & 1 & 97 \\
\hline 15 & 25 & 7 & 1 & 81 \\
\hline 16 & 27 & 5 & 1 & 87 \\
\hline 17 & 15 & 11 & 7 & 62 \\
\hline 18 & 27 & 5 & 1 & 87 \\
\hline 19 & 22 & 10 & 1 & 72 \\
\hline 20 & 16 & 10 & 7 & 64 \\
\hline 21 & 28 & 4 & 1 & 90 \\
\hline 22 & 25 & 7 & 1 & 81 \\
\hline 23 & 32 & 0 & 1 & 99 \\
\hline 24 & 16 & 11 & 6 & 62 \\
\hline 25 & 29 & 0 & 4 & 96 \\
\hline 26 & 29 & 3 & 1 & 94 \\
\hline 27 & 27 & 4 & 1 & 90 \\
\hline 28 & 32 & 0 & 1 & 99 \\
\hline 29 & 25 & 6 & 2 & 84 \\
\hline 30 & 24 & 7 & 2 & 81 \\
\hline 31 & 17 & 14 & 2 & 58 \\
\hline 32 & 30 & 0 & 3 & 97 \\
\hline 33 & 18 & 13 & 2 & 60 \\
\hline
\end{tabular}

Com base nas discordâncias entre a pesquisadora e os juízes e utilizando como critério de corte os índices abaixo de 50\%, levantou-se que somente as questões 7 e 10 não apresentaram boa capacidade de identificação de sinais de alterações de desenvolvimento da linguagem. $\mathrm{O}$ alto índice de concordância na avaliação pode ser justificado pelo fato de a lista de verificação apresentar questões que facilitam a identificação desses quadros.
Para a determinação das questões que melhor identificam sinais de transtornos do espectro autístico, foi utilizado o teste do Qui-Quadrado, indicado para esse estudo (Siegel, 1981). A Tabela 3 apresenta os resultados referentes à aplicação do cálculo do QuiQuadrado em todas as questões da lista de verificação para todos os participantes. 
Tabela 4 - Apresentação dos resultados do teste do Qui-Quadrado

\begin{tabular}{|c|c|c|c|c|c|c|c|c|}
\hline Q & $\begin{array}{l}\text { TEA } \\
\text { Sim }\end{array}$ & $\begin{array}{l}\text { TEA } \\
\text { Não }\end{array}$ & $\begin{array}{l}\text { TEA } \\
\text { Part. }\end{array}$ & $\begin{array}{l}\mathrm{AL} \\
\mathrm{Sim}\end{array}$ & $\begin{array}{c}\text { AL } \\
\text { Não }\end{array}$ & $\begin{array}{c}\text { AL } \\
\text { Part. }\end{array}$ & Qui-quadrado & $\begin{array}{c}\text { Valor } \\
\text { P }\end{array}$ \\
\hline 1 & 3 & 14 & 17 & 11 & 22 & 33 & 1,369 & 0,242 \\
\hline 2 & 0 & 17 & 17 & 25 & 8 & 33 & 25,758 & 0,000 \\
\hline 3 & 2 & 15 & 17 & 29 & 4 & 33 & 27,590 & 0,000 \\
\hline 4 & 2 & 15 & 17 & 9 & 24 & 33 & 1,572 & 0,210 \\
\hline 5 & 2 & 15 & 17 & 33 & 0 & 33 & 41,597 & 0,000 \\
\hline 6 & 0 & 17 & 17 & 27 & 6 & 33 & 30,237 & 0,000 \\
\hline 7 & 2 & 15 & 17 & 22 & 11 & 33 & 13,550 & 0,000 \\
\hline 8 & 3 & 14 & 17 & 27 & 6 & 33 & 19,251 & 0,000 \\
\hline 9 & 6 & 11 & 17 & 33 & 0 & 33 & 27,376 & 0,000 \\
\hline 10 & 17 & 0 & 17 & 25 & 8 & 33 & 4,906 & 0,027 \\
\hline 11 & 0 & 17 & 17 & 19 & 14 & 33 & 15,787 & 0,000 \\
\hline 16 & 17 & 0 & 17 & 5 & 28 & 33 & 32,782 & 0,000 \\
\hline 20 & 3 & 14 & 17 & 11 & 22 & 33 & 1,369 & 0,242 \\
\hline 24 & 17 & 0 & 17 & 26 & 7 & 33 & 4,193 & 0,041 \\
\hline 30 & 6 & 11 & 17 & 30 & 3 & 33 & 17,214 & 0,000 \\
\hline 31 & 9 & 8 & 17 & 28 & 5 & 33 & 5,937 & 0,015 \\
\hline 32 & 9 & 8 & 17 & 33 & 0 & 33 & 18,487 & 0,000 \\
\hline 33 & 11 & 6 & 17 & 20 & 13 & 33 & 0,080 & 0,777 \\
\hline
\end{tabular}

O teste do Qui-Quadrado avaliou a validade do conteúdo e, segundo o resultado do mesmo, as questões 1, 4, 20 e 33 deveriam ser retiradas da lista de verificação, por não serem significativas para a distinção entre os quadros de transtornos do espectro autístico e de alterações no desenvolvimento da linguagem, quando se está utilizando a comunicação e a linguagem como referenciais. São eles os seguintes itens: identifica letras e números (presente no CARS/ABC); utiliza marcadores de polidez - "por favor", "dá licença”, "obrigado" (presente no CARS/ADOS); identifica e nomeia marcas, objetos, rótulos, slogans (presente no CARS/ADOS) e produz imitações (presente no CHAT/ADOS/ADI-R).

Durante a realização do estudo, verificou-se que não houve diferenças significativas entre os juízes, nas duas etapas de coleta de dados, com relação à concordância nas alternativas de diagnóstico escolhidas. O alto índice de concordância confirma a hipótese de que é possível se pensar num conjunto de indicadores, no desenvolvimento da linguagem, que possam auxiliar na identificação de sinais de transtornos do espectro autístico.

A confiabilidade das alternativas escolhidas como diagnóstico foi analisada mediante a aplicação do índice de concordância Kappa (Farias Jr. Pires \& Lopes, 2002). Os valores de $\mathrm{K}$ encontrados indicaram um grau de concordância de forte a quase perfeita. Comparando-se o sim e o não da pesquisadora e dos juízes, com relação ao diagnóstico atribuído aos participantes durante a avaliação realizada na primeira etapa, foi obtido um índice de concordância kappa= $88,6 \%$ (quase perfeito).

Quando verificado o índice de concordância Kappa, entre o sim e o não da pesquisadora e dos juízes, com relação ao diagnóstico atribuído aos participantes durante a avaliação realizada na segunda etapa, obtevese um índice de concordância Kappa forte $(76,9 \%)$.

A Tabela 5 apresenta o índice de concordância Kappa dos juízes, com relação ao diagnóstico atribuído aos participantes durante as avaliações realizadas nas duas etapas do estudo. O índice de concordância Kappa observado é forte. 
Tabela 5 - Índice de concordância Kappa, para as duas etapas, comparando a concordância e discordância entre os juízes

\begin{tabular}{cccccc}
\hline & & \multicolumn{3}{c}{$\mathbf{2}^{\mathbf{a}}$ Avaliação } \\
\hline $\mathbf{1}^{\mathbf{a}}$ Avaliação & & Sim & Não & Total \\
& Sim & 13 & 5 & 18 \\
& Não & 4 & 38 & 42 \\
\cline { 2 - 5 } & Total & 17 & 43 & 60 \\
\hline
\end{tabular}

Indice de concordância kappa $=63,7 \%$

Segundo Fletcher (1996), os testes sensíveis são úteis nas fases iniciais do levantamento de uma hipótese diagnóstica, quando um grande número de possibilidades está sendo considerada e se quer reduzilas. A sensibilidade é definida como a proporção dos indivíduos com o transtorno que têm um teste positivo para o mesmo.

Já os testes específicos, são úteis para confirmar (ou incluir) um diagnóstico sugerido por outros dados. Isso porque um teste altamente específico é raramente positivo na ausência do transtorno, isto é, dá poucos resultados falso-positivos. Testes altamente específicos são particularmente necessários quando os resultados falso-positivos podem prejudicar o paciente. Especificidade é, então, a proporção dos indivíduos sem o transtorno que têm um teste negativo. Um teste específico raramente classificará erroneamente as pessoas.

A Tabela 6 apresenta a identificação positiva realizada pelos juízes para os transtornos do espectro autístico, na primeira etapa do estudo, na atribuição dos diagnósticos, após aplicação da lista de verificação pela pesquisadora. Aplicando as definições de sensibilidade e especificidade nesse grupo, podemos verificar que dos diagnósticos atribuídos ao Grupo A de transtornos do espectro autístico, dezoito foram positivos e dois negativos - uma sensibilidade de $90 \%$. Por outro lado, quarenta participantes não apresentavam as características dos transtornos do espectro autístico, a um tendo sido atribuído o diagnóstico positivo e aos outros trinta e nove o negativo, apresentando uma especificidade de 97,5\%.

Tabela 6 - Identificação positiva feita pelos juízes, para os transtornos do espectro autístico, na primeira etapa

\begin{tabular}{|c|c|c|c|c|}
\hline & & \multicolumn{3}{|c|}{ TEA } \\
\hline & & Presença & Ausência & Total \\
\hline & Positivo & 18 & 1 & 19 \\
\hline $\mathrm{J}$ & & & & \\
\hline $\mathrm{U}$ & Negativo & 2 & 39 & 41 \\
\hline Z & & & & \\
\hline $\mathrm{E}$ & Total & 20 & 40 & 60 \\
\hline $\mathrm{S}$ & & & & \\
\hline
\end{tabular}

O teste de Mann-Withney (Siegel, 1981), foi aplicado para verificar a existência de uma diferença significativa entre as concordâncias da pesquisadora e dos juízes, nos dois grupos. O resultado foi o valor $\mathrm{p}$ maior que o nível de significância adotado (nível de significância $=0,05)$, verificando-se assim que não existiu diferença significativa entre o nível de concordância da pesquisadora e dos juízes.

Numa etapa posterior, os dados obtidos na aplicação da lista de verificação foram comparados com os dados obtidos na aplicação do Autism Behavior Checklist (ABC), nos participantes dos dois grupos (A e B). Dessa forma, pretendeu-se investigar se a lista de verificação, quando comparada ao ABC, apresentava resultados que confirmassem sua utilização como instrumento que pode auxiliar na identificação de sinais de transtornos do espectro autístico.

Observou-se, então, que no GA, tanto a lista de verificação, que explora aspectos específicos do desenvolvimento da comunicação e da linguagem, quanto o Autism Behavior Checklist (ABC), que avalia aspectos mais abrangentes do desenvolvimento, apresentaram índices de 100\% de identificação de sinais de transtornos do espectro autístico. Desse 
modo, ambos foram capazes de identificar os portadores de TEA que já haviam recebido diagnóstico específico e que fizeram parte deste estudo.

No GBobservou-se que, na aplicação da lista de verificação, todos os sujeitos foram identificados como portadores de alterações de linguagem, enquanto na aplicação do Autism Behavior Checklist (ABC), nove participantes foram identificados como duvidosos para o autismo $(26,45,48,49,50,52,54,56$ e 57$)$, enquanto os outros trinta e um foram classificados como não-autistas. No estudo de Fernandes e Miilher (2008), os resultados indicaram a existência de correlações significativas entre a pontuação no $A B C$ e os resultados dos perfis funcionais da comunicação, com exceşão da subescala de linguagem, o que fez com que sugerissem que $\mathrm{O} A B C$ não deve ser utilizado como único recurso para o diagnóstico dessa população.

Os perfis de classificação desses nove participantes também sugerem que algumas características da linguagem, nos distúrbios mais severos, podem ser apresentadas por sujeitos com transtornos do espectro autístico. Uma explicação possível para esses resultados vai depender da distinção entre alguns tipos de inadequações, que anteriormente não pareciam linguisticamente explicáveis. Nos estudos de Bishop (1989) verifica-se a possibilidade de identificação de alterações cognitivas a partir de manifestações linguísticas, ou seja, a anormalidade pode não estar na forma como a mensagem é construída, mas na própria mensagem.

\section{À guisa de conclusão}

Os dados deste estudo piloto indicam que a lista de verificação proposta pode auxiliar na identificação de prováveis transtornos do espectro autístico tanto quanto o Autism Behavior Checklist (ABC), e sua especificidade para a avaliação de alterações específicas do desenvolvimento da comunicação e da linguagem, nesses casos, mostrou-se viável. Os outros instrumentos utilizados como referência para a criação da lista, como já foi citado não visam à realização de uma avaliação aprofundada e específica desses aspectos, possibilitando a diferenciação de outros quadros o que muitas vezes origina confusões durante o próprio encaminhamento para avaliação.

Considerando a Lista de Verificação, pode-se observar que os itens que a compõem objetivam avaliar o desenvolvimento de diferentes aspectos da comunicação e da linguagem presentes ou ausentes nos transtornos do espectro autístico, e Fernandes (1996) afirmou que, independentemente do conceito, da etiologia e do critério diagnóstico envolvendo o autismo infantil, a linguagem sempre representa um aspecto fundamental desse quadro clinico.

A identificação e avaliação de prejuízos à comunicação e à linguagem, por serem aspectos que geralmente estão presentes desde o início, podem auxiliar no levantamento da hipótese diagnóstica desses transtornos e no encaminhamento para avaliações mais abrangentes, bem como para atendimentos mais adequados e que poderão influenciar no prognóstico dos casos.

Salienta-se que foram muitas as dificuldades encontradas para realização deste estudo piloto, principalmente as referentes ao acesso à população relacionada à pesquisa, ao tempo que se demorou em fazer o levantamento de uma hipótese diagnóstica para os grupos participantes e ao encaminhamento para o atendimento adequado, o que ressalta a importância de pesquisas que visem à construção e validação de instrumentos que possam auxiliar nessa avaliação, tornado-a mais rápida e acessível.

Quando essas dificuldades foram superadas e a pesquisa foi desenvolvida, a lista de verificação aqui apresentada mostrou-se um instrumento viável para auxiliar na avaliação dos transtornos do espectro autístico e na sua possível diferenciação de quadros relacionados às alterações do desenvolvimento da linguagem. A forma como a mesma foi estruturada para aplicação pode viabilizar seu uso em serviços públicos, clínicas e instituições, inclusive por profissionais sem experiência nessa área específica, bem como pode auxiliar na diminuição do tempo entre uma primeira avaliação e o encaminhamento adequado para avaliações mais aprofundadas e particulares. Entretanto, aponta-se também a necessidade de realização de outros estudos que possam utilizar a lista de verificação em um maior número de sujeitos, observando melhor as especificidades e resultados aqui apresentados, com a finalidade de corroborá-los ou não, levando-se em consideração todas as limitações envolvidas nesse primeiro estudo.

\section{Referências}

Amato, C. A. I. H. (2000). Estudo comparativo dos processos de aquisição da linguagem não verbal em crianças pré verbais autistas e normais. (Dissertação de Mestrado). Universidade de São Paulo, Faculdade de Medicina, São Paulo, Brasil.

Baron-Cohen, S. (1990). Autism, a specific cognitive disorder "mindblindness". International Journal of Psychiatric, 2, 81-90. 
Baron-Cohen S. (1991). The development of a theory of mind in autism: deviance an delay? Psychiatric Clinical North American, 14(1), 33-52.

Baron-Cohen, S. (1988). Social and pragmatic deficits in autism: cognitive or affective? Journal Autism Development Disorders, 18(3), 379-401.

Bates, E., Camaioni, L. \& Volterra, V. (1976). The acquisition of performatives prior to speech. Em: E. Battes. (Org.). Language and Context. (pp. 11128). New York: Academic Press.

Bateson, M. C. (1979). The epigenesist of conversational interaction: a personal account of research development. Em: M. Bullowa. (Org.). Before Speech: the beginning of interpersonal communication. (pp. 63-78). Cambridge University Press.

Befi-Lopes, D. \& Cáceres, A. M. (2010) Language profiles in Autism Spectrum Disorders (ASD), Specific Language Impairment (SLI) and Attention Deficit Hyperactivity Disorder (ADHD). Revista da Sociedade Brasileira de Fonoandiologia, 15(2), 305-306.

Bishop, D. V. M. (1989). Autism, Asperger's syndrome and semantic-pragmatic disorder: where are the boundaries? British Journal of Disorders of Communication, 24, 107-21.

Blomm, L. \& Lahey, M. (1988). Language disorders and language development. New York: Macmillan.

Brazelton, T. B. (1979). Evidence of communication during neonatal behavior assessment. Em: M. Bullowa. (Org.). Before Speech: the beginning of interpersonal communication. (pp. 79-88). Cambridge University Press.

Bruner, J. S. (1975). The ontogenesis of speech acts. Journal Child Language, 2, 1-9.

Bruner, J. S. (1978). From communication to language a psychological perspective. Em: M. Markova. (Org.). The social contexto of language. (pp. 17-48). Chicester, Wiley \& Sons.

Bullowa, M. (1979). Before Speech: the beginning of interpersonal communication. Cambridge: Cambridge University Press.

Carvalho, L. R. L., Mecca, F. F. N. \& Lichtig, I. (2008). Avaliação das habilidades de metarrepresentação em crianças de sete a oito anos. Pró-Fono Revista de Atualização Científica, 20(2), 81-86.

Chappell, P. F. \& Sander, L. W. (1979). Mutual regulation of neonatal interactive process: context for de origins of communication. Em: M.
Bullowa. (Org.). Before Speech: the beginning of interpersonal communication. (pp. 89-110). Cambridge: Cambridge University Press.

Farias Jr., J. C., Pires, M. C. \& Lopes, A. S. (2002). Reprodutibilidade de um questionário para $\mathrm{O}$ levantamento de informações sobre comportamentos relacionados à saúde em adolescentes. Revista. Brasileira de Ciências e Movimento, Brasília, 10(3), 43-8.

Farrell, M. (2008). Dificuldades de comunicação e autismo: guia do professor. Porto Alegre, Artmed.

Fernandes, F. D. M. (1995). Aspectos funcionais da comunicação de crianças com sindrome autística. (Tese de Doutorado). Universidade de São Paulo, Faculdade de Filosofia, Letras e Ciências Humanas, São Paulo, São Paulo, Brasil.

Fernandes, F. D. M. (1996). Autismo infantil: repensando o enfoque fonoaudiológico. São Paulo: Lovise.

Fernandes, F. D. M. (1998). Os atrasos de aquisição de linguagem. Em: M. Goldfeld. (Org.). Fundamentos em fonoaudiologia: linguagem. (pp. 22-37). Rio de janeiro: Guanabara Koogan.

Fernandes, F. D. M. (2000a). Aspectos funcionais da comunicação de crianças autistas. Transtornos do Desenvolvimento, São Paulo, 9(51), 25-35.

Fernandes, F. D. M. (2000b). Sistematização de dados referentes à atuação fonoaudiológica em hospitaldia infantil: o perfil comunicativo como indicador de desempenho. Pró-Fono Revista de Atualização Cientifica, 12(1), 1-8.

Fernandes, F. D. M. (2002). Atuação fonoaudiológica com crianças com transtornos do espectro autístico. (Tese de Livre Docência). Universidade de São Paulo, Faculdade de Medicina, São Paulo, São Paulo, Brasil.

Fernandes, F. D. M., \& Miilher, L. P (2008). Relações entre a Autism Behavior Checklist (ABC) e o perfil funcional da comunicação no espectro autístico. Pró-Fono Revista de Atualização Científica, 20(2), 111-116.

Fletcher, R. H., Fletcher, S. W. \& Wagner, E. H. (1996). Epidemiologia clínica: elementos essenciais $\left(3^{\mathrm{a}}\right.$ Ed.). Tradução Bruce B. Duncan, Maria Inês Schimidt Porto Alegre: Artes Médicas.

Frith, U. (1988). Autism: possible clues to the underlyng pathology. Psychological facts. Em: L. Wing. (Org.). Aspects of autism: biological research. London: (pp. 1-10). Gaskel Eds. \& Royal College of Psychiatrists \& The National Autistic Society. 
Frith, U. (1989). Autism, explaining the enigma. Oxford: Blackwell Pub.

Harding, C. G. (1983) Setting the stage for language acquisition: communication development in the first year. Em: R. M. Golinkoff. (Org.). The transition from prelinguistic to linguistic communication. New Jersey: L.E.A.

Kanner, L. (1943). Autistic disturbances of affective contact. Nervous Child, Baltimore, 2, 217-250.

Marteleto, M. R. F. (2003). Validade e confiabilidade da escala de comportamentos autisticos (ABC): estudo preliminar. (Dissertação de Mestrado). Faculdade de Ciências da Saúde da Escola Paulista de Medicina, São Paulo, São Paulo, Brasil.

Molini-Avejonas, D. R. \& Fernandes, F. D. M. (2004). Alterações pragmáticas, cognitivas e sociais em crianças com autismo: revisão de literatura. R. Soc. Bras. Fonoaudiol., São Paulo, 9(3), 179-186.
Ochs Keenan E., Schieffelin B. (1983) Topics as a discourse notion: a study of topic in the conversations of children and adults. Em: E. Ochs Keenan, B. Schieffelin (Orgs.). Acquiring conversational competence. (pp. 66-113). London: Routledge \& Kegan Paul.

Siegel, S. (1981). Estatística não-paramétrica para as ciências do comportamento. Tradução Alfredo Alves de Faria, São Paulo: Mc Graw Hill do Brasil.

Tamanaha, A .C., Perissinoto, J. \& Chiari, B. M. (2008). Uma breve revisão histórica sobre a construção dos conceitos o autismo infantil e da síndrome de asperger. Revista da Sociedade Brasileira de Fonoaudiologia, 13(3), 296-299.

Sobre as autoras:

Silvana Batista Gaino é psicóloga, mestre em Semiótica e Lingüística Geral pela Universidade de São Paulo e professora assistente da Universidade Federal do Recôncavo da Bahia.

Manoel Henrique Salgado é matemático, professor assistente e doutor da Universidade Estadual Paulista Júlio de Mesquita Filho, UNESP-Bauru/SP.

Fernanda Dreux Miranda Fernandes é fonoaudióloga, professora livre-docente do Departamento de Fisioterapia, Fonoaudiologia e Terapia Ocupacional da Faculdade de Medicina da Universidade de São Paulo e foi orientadora da dissertação de mestrado que deu origem a este artigo. 
